\title{
Intelligent Autonomous Systems: A Layered Architecture in the Age of Multicore Processing
}

\author{
Guna Seetharaman \\ Information Directorate, Air Force Research Laboratory, Rome, NY, USA \\ guna@cacs. louisiana. edu
}

\begin{abstract}
Research in intelligent autonomous systems has historically been constrained by access to scalable high-performance computing. The challenges associated with scalability and tractability have significantly influenced analytical approaches and may have led to analysis in the lower dimensional spaces. Such reductions may overly simplify approaches to model and exploit redundancy in manners that human perception is able to in a bidirectional inferencing process. Evidence indicates that human perception involves different methods of classifying, indexing and associating information according to different spatio-temporal and saliency metrics. Recent surge in high performance computing with multicore processors and wide spread access to large disk-space at finer granularity, have triggered a renewed assessment of existing approaches. A survey of the techniques lead to a compelling need for efficient methods for capturing and exploiting "context" and "context-specific information" to swiftly change the behaviors of intelligent systems. The talk will highlight the evolution of signal processing, linguistic hierarchy, computing models where information fusion is considered, and draw some parallels, to make a case for a layered architecture for context-adaptive autonomous systems. Interactive access to petascale supercomputing has given a newer framework to incorporate context in such studies at scale that is required to perform complex intelligence tasks. The talk will highlight our current research in this direction including our vision of the future of intelligent autonomous systems with embedded high-performance computing with reach back compute power.
\end{abstract}

